\title{
Telehealth Training Is Essential to Care for Underserved Populations: a Medical Student Perspective
}

\author{
Stephanie S. Camhi ${ }^{1}$ (D) $\cdot$ Alexandra Herweck ${ }^{1}$ (D) $\cdot$ Hanna Perone ${ }^{1}$ (D) \\ Published online: 15 June 2020 \\ (C) International Association of Medical Science Educators 2020
}

\begin{abstract}
Telehealth is an area of medicine which has magnified the ability to treat patients remotely. Presently the education of medical professionals pertaining to the value, use, and implementation of telehealth is not adequate to harness the potential of available technologies. Patients engaging in telehealth experience time and cost savings, improved disease management through remote monitoring programs, and high-quality care regardless of geographic location. Despite this, medical education has been slow to evolve. It is therefore imperative that medical curricula incorporate training for this rapidly advancing mode of healthcare delivery to enable students to best care for their future patient population.
\end{abstract}

Keywords Telemedicine $\cdot$ Telehealth $\cdot$ e-health $\cdot$ Preclinical medical education

Telehealth (telemedicine, e-health) refers to utilization of technology to deliver healthcare services outside of traditional healthcare facilities such as through remote monitoring, video-conferencing, or electronic consultations [1]. Surveys from the American Hospital Association support that use of telehealth has grown dramatically in the past decade, with $76 \%$ of United States (US) hospitals offering some form of telehealth services [2]. While thirty-five states in the US currently have enacted parity laws requiring health insurers to reimburse telehealth services, Medicare reimbursement is slow to provide adequate compensation to all involved parties [2]. The advent of the coronavirus disease 2019 (COVID-19) global pandemic propelled telehealth to the forefront with virtual care visits predicted to surpass 1 billion in this calendar year [3, 4]. While the agility of the healthcare system to adapt to this unprecedented challenge is admirable, the retroactive application of telehealth approaches highlights how unprepared our health system is to embrace widespread telehealth implementation. In part, this may be related to the fact that medical schools are similarly slow to adopt the growing movement of telehealth despite its promotion by the

Stephanie S. Camhi

stephanie.camhi@med.miami.edu

1 MD/MPH Program, University of Miami Miller School of Medicine, 1600 NW 10th Ave \#1140, Miami, FL 33136, USA
American Medical Association (AMA). Telemedicine has significant potential to combat health disparities, improve access to healthcare across all specialties, including the care of stigmatized populations, and improve patient outcomes through remote monitoring programs; however, effective implementation by healthcare providers requires early exposure throughout both allopathic and osteopathic education to adequately harness this potential.

As of 2016, less than half of allopathic medical programs in New York and Texas reported implementing preclinical telemedicine education. In the same time frame, states with predominantly rural populations, namely North Dakota, Kansas, and Oklahoma, had yet to implement preclinical telemedicine in any of their allopathic schools [5]. According to the Association of American Medical Colleges (AAMC), the number of medical schools offering clerkship electives in telemedicine has increased from 44 in 2013 to 68 in 2019 [6]. While this increase is promising, the elective nature of telehealth training remains concerning. The AMA seems to share this sentiment and recently launched the "Accelerating Change in Medical Education Consortium" which granted $\$ 12.5$ million to 32 medical schools for telehealth curricular development [7]. It is worth noting that medical education already aims to deliver a surplus of crucial biomedical information on a rather accelerated timeline, which appears to push telemedicine to the hierarchical backburner. One study from Australia highlighted that telehealth training is not a priority for medical students given that accrediting bodies do not 
expect competency in this domain from medical graduates [8]. However, with the advent of the COVID-19 pandemic, and despite the fact that mandatory telemedicine education is currently lacking in most allopathic schools, the United States Medical Licensing Examination (USMLE) has suggested that a telemedicine-style clinical skills examination may be forthcoming. This presents additional challenges including the complexity of technical work required for initiation of telehealth education, which to this point has been lacking, as well as the concerns of standardization and validation of new assessments over electronic video platforms [9]. Despite these challenges, a few preliminary studies suggest that curricular reform to introduce and instruct regarding telemedicine is likely to be promising. One study from the University of Iowa implemented a 3-module introductory educational program for second-year students and improved students' telemedicine knowledge and confidence [10]. A study from the Uniformed Services University of the Health Sciences similarly demonstrated an increase in telehealth knowledge following student completion of their pilot program consisting of telemedicine training, faculty-supervised patient encounters, and an introduction to telehealth surgical equipment [11].

Telehealth applications stand to benefit patients given that medical appointments cause hourly workers to lose valuable income through loss of time at work, personal transportation fees, and copayments or other associated visit costs. Recent work by Ray et al. found the total time invested in travel to and from, wait time associated with, and clinical interaction comprising a single appointment to be 121 minutes [12] After this substantial time and monetary investment, the patient may not even be visiting a center that is specialized in treatment of their specific complaint, leading to receipt of suboptimal medical care. Another study at Vermont Veterans Hospital showed an average travel and cost savings of 145 miles and 142 minutes per visit with the use of telemedicine clinic appointments [13].

In addition to permitting time and cost savings, telemedicine is essential to bridge structural barriers and provide access to vital emergency and/or specialty care to individuals in under-resourced settings. Utilization of telehealth for triage has shown to be acceptable to patients and to potentially reduce the number of emergency department (ED) visits [14]. Telehealth approaches also show promise for providing crucial urgent care services to rural populations [15]. This reduction in structural barriers does not come at a cost to patient satisfaction. A study in oncology patients suggested equivalent satisfaction ratings regardless of the care delivery method including telephone versus in-person appointments [16]. Furthermore, telemedicine has improved access to specialists in resource-poor communities. One study of an interactive video colposcopy initiative in rural Arkansas allowed health department nurses to conduct colposcopies and cervical biopsies through real-time inclusion of an obstetric-gynecologic specialist [17]. Similarly, dermatology and gastroenterology telehealth pilot programs have decreased time to initiation of patient care [18]. During the COVID-19 pandemic, telemedicine has been harnessed to continue regular non-urgent outpatient care and to provide COVID-related care such as symptomatic monitoring and post-discharge follow-up [3]. Widespread use and availability of mobile technologies allow for telemedicine utilization throughout the country. As of $2018,81 \%$ of Americans own a smartphone, nearly $75 \%$ of adults own desktop or laptop computers, and approximately $50 \%$ own tablet computers [19]. More than ever, individuals from all racial, ethnic, and income backgrounds can afford these technologies and therefore already possess the necessary supplies to engage in telehealth services.

In the last 15 years, remote patient monitoring (RPM) via noninvasive devices has gained popularity and increased in availability for collection of immediate medical information and thereby monitoring of chronic health conditions. The most common RPM devices used include smartphones (19\%), wearable devices (18\%), biosensor devices (11\%), and computerized systems (10\%), with most RPM programs including multiple methodologies of monitoring patients (42\%) [20]. RPM programs have improved self-management, which has been shown to reduce clinic visits and hospitalizations, for patients with chronic disease and reduced the severity of symptoms related to chronic respiratory, metabolic, and cardiovascular conditions [20]. Patients with type 2 diabetes mellitus in an RPM program lost weight and had positive A1c outcomes [21]. In a study utilizing RPM for blood pressure control, systolic blood pressure outcomes were similar in patients with RPM alone compared with those who received both RPM and regular office visits, suggesting that both approaches were as efficacious as usual office care [22].

Telehealth is also an important avenue for providing care to populations who may otherwise be stigmatized. Men who have sex with men (MSM) face many structural and cultural barriers, and studies have shown worse health outcomes in this population, particularly in those with HIV or AIDS. A recent study of MSM in Oklahoma suggested that geographical isolation significantly limits access to quality sexual healthcare and resources including HIV pre-exposure prophylaxis (PrEP) [23]. A pilot study in the rural US showed that electronic prescribing of PrEP resulted in many individuals initiating treatment, and additionally was preferred by patients over standard in-person care [24]. In addition to PrEP availability, MSM have also indicated acceptability of telemedicine efforts to increase sexually transmitted infection (STI) screening. In a pilot study of home sampling for STI screening, forty-nine of fifty enrolled MSM couples completed testing, indicating high acceptance among the MSM population for this model [25].

Telehealth further stands to improve ease of access to female reproductive health services including oral 
contraception. As of February 2018, nine platforms were electronically prescribing oral contraception with a greater adherence to $\mathrm{CDC}$ prescribing guidelines compared with in-person office visits [26, 27]. Tele-contraception efforts have not only increased access to oral contraception but also empowered women with adequate knowledge pertaining to their contraceptive options [28]. These are merely a handful of studies highlighting the immense power for telemedicine to improve healthcare accessibility in stigmatized populations and promote safe sexual health practices.

Delivery of the highest quality telehealth care relies on physicians from numerous specialties, and therefore, all osteopathic and allopathic students would benefit from early exposure and training. There is much conversation in the medical education community pertaining to curricular reform and training "Physicians of the Future." While these groups discuss shortening the preclinical experiences, integrating basic sciences into clinical studies, and transitioning to symptombased education, an emphasis on telehealth is generally lacking. Greater urgency should be exhibited to incorporate telemedicine into the training of young physicians and thereby harness the full potential of our rapidly advancing medical technologies. Introduction specifically at the medical student level will provide students with a framework for developing further telehealth knowledge and experience as they progress through training. Early and repeated exposure across varying institutions (i.e., medical school, residency training, fellowship training) will provide a more thorough "tour" of available telehealth approaches and technologies. In addition, the initial clerkship year, or just prior, is an ideal time frame for introduction given that students will then feel as though telemedicine is engrained into their clinical workflow.

Medical students in Germany identified the growing prevalence of telehealth applications as an important learning goal [29]. A mixed-methods review of telehealth applications in US allopathic schools similarly details that most medical students find telemedicine-based training to be valuable [5]. Telehealth applications are a main reason that the COVID19 pandemic failed to bring our medical education to a startling halt. Objective structured clinical examinations (OSCEs) were adjusted to take place as telemedicine encounters over video conference for core clerkships including pediatrics and psychiatry. In addition, a medical student-driven initiative to offer telehealth education to clerkship students, consisting of an introductory lecture and participation in telehealth encounters, was mandated for all 200 third-year students at our institution. Following the lecture, students were paired with a provider in their specialty area of interest to participate in telehealth encounters. These experiences provide just a small taste of what telehealth applications stand to contribute to our future toolkit as physicians, and similar activities should be built into the curriculum rather than mandated in the context of pandemic response.
Some of the barriers to widespread implementation of telemedicine include uncertainty pertaining to reimbursement and licensure requirements. Recent governmental actions have lessened these concerns, leaving the lack of training of medical professionals as a key barrier to implementation [5]. Without expedited implementation of training within undergraduate medical education, the likelihood of provider knowledge keeping up with rapid technological advances is slim. How will we become public health physicians of the future without this vital training? How will we bridge the inequities that structural barriers have enacted? How will we offer the best care, to all patients, regardless of gender, race, religion, stigmatization of their medical condition, or physical location? And perhaps most pressingly, how can we ensure that our pandemic response is not dampened by the learning curve associated with retroactive telemedicine implementation? The answer is that we, as current physicians-in-training, will not succeed in these endeavors if our medical education does not prepare us to do so. Our inability, as care providers, to implement telemedicine is a disservice to the rural, structurally disadvantaged, impoverished, and stigmatized communities which we, as physicians, cannot morally afford to underserve.

Acknowledgments The authors are thankful to Dr. Daniel Lichtstein for his thoughtful review and support of our work.

Code Availability Not applicable.

Authors' Contributions All authors made substantial contributions to the conception of the work, drafted the work and revised it critically for important intellectual content, and approved the version to be published.

Availability of Data and Material Not applicable.

\section{Compliance with Ethical Standards}

Conflict of Interest The authors declare that they have no conflict of interest.

\section{References}

1. WHO | Telehealth. WHO. http://www.who.int/sustainabledevelopment/health-sector/strategies/telehealth/en/. Accessed 6 Feb 2020.

2. fact-sheet-telehealth-2-4-19.pdf. https://www.aha.org/system/files/ 2019-02/fact-sheet-telehealth-2-4-19.pdf. Accessed 6 Feb 2020.

3. Wosik J, Fudim M, Cameron B, Gellad ZF, Cho A, Phinney D, et al. Telehealth transformation: COVID-19 and the rise of virtual care. J Am Med Inform Assoc. Published online April 20, 2020. https://doi.org/10.1093/jamia/ocaa067.

4. Portnoy J, Waller M, Elliott T. Telemedicine in the era of COVID19. J Allergy Clin Immunol Pract. 2020;8(5):1489-91. https://doi. org/10.1016/j.jaip.2020.03.008.

5. Waseh S, Dicker AP. Telemedicine training in undergraduate medical education: mixed-methods review. JMIR Med Educ. 2019;5(1). https://doi.org/10.2196/12515. 
6. Content Documentation in Required Courses and Elective Courses. AAMC. https://www.aamc.org/data-reports/curriculum-reports/ interactive-data/content-documentation-required-courses-andelective-courses. Accessed 6 Feb 2020.

7. AMA encourages telemedicine training for medical students, residents. American Medical Association. https://www.ama-assn.org/ press-center/press-releases/ama-encourages-telemedicine-trainingmedical-students-residents. Accessed Feb 62020.

8. Edirippulige S, Brooks P, Carati C, Wade VA, Smith AC, Wickramasinghe $\mathrm{S}$, et al. It's important, but not important enough: eHealth as a curriculum priority in medical education in Australia. $\mathrm{J}$ Telemed Telecare. 2018;24(10):697-702. https://doi.org/10.1177/ $1357633 X 18793282$.

9. United States Medical Licensing Examination $\mid$ Announcements. Accessed May 28, 2020. https://www.usmle.org/announcements/? ContentId $=284$

10. Walker C, Echternacht H, Brophy PD. Model for medical student introductory telemedicine education. Telemed J E Health. 2019;25(8):717-23. https://doi.org/10.1089/tmj.2018.0140.

11. Jonas CE, Durning SJ, Zebrowski C, Cimino F. An interdisciplinary, multi-institution telehealth course for third-year medical students. Acad Med. 2019;94(6):833-7. https://doi.org/10.1097/ ACM.0000000000002701.

12. Opportunity Costs of Ambulatory Medical Care in the United States. AJMC. https://www.ajmc.com/journals/issue/2015/2015vol21-n8/opportunity-costs-of-ambulatory-medical-care-in-theunited-states. Accessed 6 Feb 2020.

13. Russo JE, McCool RR, Davies L. VA telemedicine: an analysis of cost and time savings. Telemed J E Health. 2016;22(3):209-15. https://doi.org/10.1089/tmj.2015.0055.

14. van Veen T, Binz S, Muminovic M, et al. Potential of mobile health technology to reduce health disparities in underserved communities. West J Emerg Med. 2019;20(5):799-802. https://doi.org/10. 5811/westjem.2019.6.41911.

15. Khairat S, Haithcoat T, Liu S, et al. Advancing health equity and access using telemedicine: a geospatial assessment. J Am Med Inform Assoc. 2019;26(8-9):796-805. https://doi.org/10.1093/ jamia/ocz108.

16. Sprague SL, Holschuh C. Telemedicine versus clinic visit: a pilot study of patient satisfaction and recall of diet and exercise recommendations from survivorship care plans. Clin J Oncol Nurs. 2019;23(6):639-46. https://doi.org/10.1188/19.CJON.639-646.

17. Lowery CL, Bronstein JM, Benton TL, Fletcher DA. Distributing medical expertise: the evolution and impact of telemedicine in Arkansas. Health Aff (Millwood). 2014;33(2):235-43. https://doi. org/10.1377/hlthaff.2013.1001.

18. Holmes AN, Chansky PB, Simpson CL. Teledermatology consultation can optimize treatment of cutaneous disease by nondermatologists in under-resourced clinics. Telemed J E Health. 2019. https://doi.org/10.1089/tmj.2019.0217.
19. NW 1615 L. St, Suite 800Washington, Inquiries D 20036USA202419-4300 | M-857-8562 |F-419-4372 | M. Demographics of Mobile Device Ownership and Adoption in the United States. Pew Research Center: Internet, Science \& Tech. https://www. pewresearch.org/internet/fact-sheet/mobile/. Accessed 6 Feb 2020.

20. Vegesna A, Tran M, Angelaccio M, Arcona S. Remote patient monitoring via non-invasive digital technologies: a systematic review. Telemed J E Health. 2017;23(1):3. https://doi.org/10.1089/ tmj.2016.0051.

21. Michaud TL, Siahpush M, Estabrooks P, et al. Association between weight loss and glycemic outcomes: a post hoc analysis of a remote patient monitoring program for diabetes management. Telemed $\mathrm{J} \mathrm{E}$ Health. 2019. https://doi.org/10.1089/tmj.2019.0030.

22. Kim Y-N, Shin DG, Park S, Lee CH. Randomized clinical trial to assess the effectiveness of remote patient monitoring and physician care in reducing office blood pressure. Hypertens Res. 2015;38(7): 491-7. https://doi.org/10.1038/hr.2015.32.

23. Hubach RD, Currin JM, Sanders CA, et al. Barriers to access and adoption of pre-exposure prophylaxis for the prevention of HIV among men who have sex with men (MSM) in a relatively rural state. AIDS Educ Prevent. 2017;29(4):315-29. https://doi.org/10. 1521/aeap.2017.29.4.315.

24. Siegler et al. PILOT TEST OF A PrEP TELEMEDICINE SYSTEM FOR YOUNG BLACK MSM IN THE RURAL US SOUTH | CROI Conference. http://www.croiconference.org/ sessions/pilot-test-prep-telemedicine-system-young-black-msmrural-us-south. Accessed 6 Feb 2020.

25. Sullivan S, Sullivan P, Stephenson R. Acceptability and feasibility of a telehealth intervention for sexually transmitted infection testing among male couples: protocol for a pilot study. JMIR Res Protoc. 2019;8(10):e14481. https://doi.org/10.2196/14481.

26. Zuniga C, Grossman D, Harrell S, Blanchard K, Grindlay K. Breaking down barriers to birth control access: an assessment of online platforms prescribing birth control in the USA. J Telemed Telecare. 2019:1357633X18824828. https://doi.org/10.1177/ $1357633 X 18824828$

27. A Study of Telecontraception | NEJM. https://www.nejm.org/doi/ full/10.1056/NEJMc1907545. Accessed 10 Feb 2020.

28. Sundstrom B, DeMaria AL, Ferrara M, Meier S, Billings D. "The closer, the better:" the role of telehealth in increasing contraceptive access among women in rural South Carolina. Matern Child Health J. 2019;23(9):1196-205. https://doi.org/10.1007/s10995-01902750-3.

29. Dannenberg KA, Stroben F, Schröder T, Thomas A, Hautz WE. The future of practical skills in undergraduate medical education an explorative Delphi-Study. GMS J Med Educ. 2016;33(4): Doc62. https://doi.org/10.3205/zma001061.

Publisher's Note Springer Nature remains neutral with regard to jurisdictional claims in published maps and institutional affiliations. 